\title{
Therapeutic Effect of IL1ß Priming Tonsil Derived-Mesenchymal Stem Cells in Osteoporosis
}

\author{
Minjoo Yoo' ${ }^{1}$ (D) Sungkuk Cho' ${ }^{1} \cdot$ Sunhye Shin $^{1}$ (1) Jung-Mi Kim $^{1}$ (D)

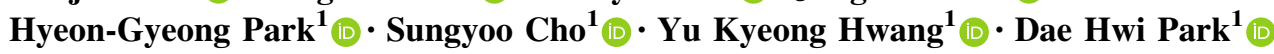

Received: 8 April 2021/Revised: 27 April 2021/Accepted: 30 April 2021/Published online: 11 June 2021

(C) The Author(s) 2021

\begin{abstract}
BACKGROUND: Stem cell therapies can be a new therapeutic strategy that may rebalance anabolic and anti-resorptive effects in osteoporosis patients. Tonsil-derived mesenchymal stem cells (TMSCs) can be an alternative therapeutic source for chronic degenerative diseases including osteoporosis. MSCs acquire immune regulatory function under the inflammatory cytokines. Since interleukin (IL) $1 \beta$ is known to be one of inflammatory cytokines involved in osteoporosis progression, treatment of IL1 $\beta$ with TMSCs may enhance immunomodulatory function and therapeutic effects of TMSCs in osteoporosis. METHODS: For IL1 $\beta$ priming, TMSCs were cultured in the presence of the medium containing IL1 $\beta$ for 1 day. Characteristics of IL1 $\beta$ priming TMSCs such as multipotent differentiation properties, anti-inflammatory potential, and suppression of osteoclast differentiation were assessed in vitro. For in vivo efficacy study, IL1 $\beta$ priming TMSCs were intravenously infused twice with ovariectomized (OVX) osteoporosis mouse model, and blood serum and bone parameters from micro computed tomography images were analyzed.

RESULTS: IL1 $\beta$ priming TMSCs had an enhanced osteogenic differentiation and secreted factors that regulate both osteoclastogenesis and osteoblastogenesis. IL1 $\beta$ priming TMSCs also suppressed proliferation of peripheral blood mononuclear cells (PBMCs) and decreased expression of Receptor activator of nuclear factor kappa-B ligand (RANKL) in PHA-stimulated PBMCs. Furthermore, osteoclast specific genes such as Nuclear factor of activated T cells c1 (NFATc1) were effectively down regulated when co-cultured with IL1 $\beta$ priming TMSCs in RANKL induced osteoclasts. In OVX mice, IL1 $\beta$ priming TMSCs induced low level of serum RANKL/osteoprotegerin (OPG) ratio on the first day of the last administration. Four weeks after the last administration, bone mineral density and serum Gla-osteocalcin were increased in IL1 $\beta$ priming TMSC-treated OVX mice. Furthermore, bone formation and bone resorption markers that had been decreased in OVX mice with low calcium diet were recovered by infusion of IL1 $\beta$ priming TMSCs.

CONCLUSION: IL1 $\beta$ priming can endow constant therapeutic efficacy with TMSCs, which may contribute to improve bone density and maintain bone homeostasis in postmenopausal osteoporosis. Therefore, IL1 $\beta$ priming TMSCs can be a new therapeutic option for treating postmenopausal osteoporosis.
\end{abstract}

Keywords Palatine tonsil $\cdot$ Mesenchymal stem cells $\cdot$ Interleukin $1 \beta \cdot$ Osteoporosis

Supplementary Information The online version contains supplementary material available at https://doi.org/10.1007/s13770021-00350-3.

Dae Hwi Park

parkdh@greencross.com

1 Cell Therapy Research Center, Green Cross LabCell, 107, Ihyeon-ro 30 beon-gil, Giheung-gu, Yongin-si, Gyeonggi-do 16924, South Korea

\section{Introduction}

Osteoporosis is a systemic bone disease characterized by reduced bone density and deterioration of micro-architecture of bone tissue. Prevalence of osteoporosis is higher in elderly population. In particular, one in three women over age 50 has higher hip fracture and mortality rates due to 
postmenopausal osteoporosis [1]. Although anti-catabolic drugs such as bisphosphonate and anabolic drugs, PTH and anti-sclerostin, are available to treat postmenopausal osteoporosis, these drugs may be used for a limited period of time in some patients due to high risk of side effects [2-5]. Furthermore, most of the drugs used for osteoporosis cannot restore bone loss in patients. Therefore, there are still clinical unmet needs for developing new drugs including stem cell therapy that not only prevents the bone loss, but also induces recovery from the bone loss by rebalancing bone homeostasis with minimum side effects [6].

Mesenchymal stem cells (MSCs) can be isolated from various tissues such as adipose tissue, bone marrow, and umbilical cord blood. MSCs have multipotent differentiation property into bone, cartilage, fat, and others. MSCs based therapy may be a good candidate for the new therapeutic options that aim for both anabolic and anti-resorptive effects on bones in osteoporosis. Bone marrow derived mesenchymal stem cells (BM-MSCs) have been used in the most stem cell based studies for osteoporosis. Since isolation of the bone marrow from a donor is an invasive procedure and yield of BM-MSC isolation from the bone marrow may not be sufficient for commercial scale manufacturing, application of MSCs from alternative sources has been investigated for osteoporosis treatment.

Accumulating evidence suggests that human tonsil derived mesenchymal stem cells (TMSCs) may have potential to regenerate damaged tissues in inflammatory disease [7, 8], endocrine disease [9, 10], and osteoporosis [11-13]. TMSCs constitutively expressed anti-inflammatory factors such as PD-L1 and interleukin-1 receptor antagonist (IL-1RA) [14]. Furthermore, conditioned medium from TMSCs attenuated Th17 mediated inflammation in psoriatic mouse model [15]. In addition, TMSCs secreted osteoprotegerin (OPG) that is able to regulate Th17induced osteoclastogenesis [16]. Based on these finding, TMSCs is likely to be an ideal source of stem cell therapy for osteoporosis, which have both anti- inflammatory function and ability to recover bone balance.

Postmenopausal osteoporosis results from lack of estrogen that may lead to an increase in pro-inflammatory cytokines and imbalance between bone resorption and bone formation [17, 18]. In pathological condition in osteoporosis, pro-inflammatory cytokines such as tumor necrosis factor alpha (TNF $\alpha$ ), IL1 $\beta$, and IL17 were increased [19-21]. A previous study demonstrated that IL1 $\beta$ induced an increase of RUNX2 expression in bone marrow stem cells and promoted differentiation into osteoblasts via Wnt5a pathway [22]. However, biological effects of systemically infused IL1 $\beta$ priming TMSCs in postmenopausal osteoporosis model have not been investigated. In this study, we first investigated whether IL1 $\beta$ priming TMSCs modulate immune cell function and osteoclast differentiation in vitro.
Subsequently, we also examined effects of intravenous infusion of IL1 $\beta$ priming TMSCs on recovery from bone loss in ovariectomized osteoporosis mouse model.

\section{Methods}

\subsection{Animal model for osteoporosis and systemic infusion of IL1 $\beta$ priming TMSCs}

6 weeks old female Balb/C mice were purchased from Orient Bio Inc. (Seongnam, Korea). All experiments were performed in accordance with guideline of Institutional Animal Care and Use Committee of GC pharma animal research center (Yong-in, Korea). Osteoporosis was induced in 7 weeks old Balb/C mice by performing ovariectomy (OVX) under general anesthesia using isoflurane (JW pharma, Seoul, Korea). For sham group mice, same surgical procedure was performed, but ovaries were not removed. After surgery, sham and OVX groups were fed low-calcium diet $(0.01 \%$ low calcium diet, TD.95027, Harlan Laboratories, Inc., Indianapolis, IN, USA). After 8 weeks, mice were intravenously injected once a month for 2 months with vesicle $(0.1 \mathrm{~mL}$ PBS) or TMSCs $\left(8 \times 10^{5}\right.$ cells in $0.1 \mathrm{~mL}$ PBS $)$. Bones were dissected and fixed in $10 \%$ neutral formalin solution (SigmaAldrich, St. Louis, MO, USA) overnight for histological analysis and micro-CT analysis.

\subsection{Culture of mesenchymal stem cells}

Tonsil tissue was provided by Prof. Han Su Kim and Prof. Soo Yeon Jung (IRB approved by Ewha Womans University Mok-Dong hospital, Seoul, Korea; no. ECT 11-53-02). Isolation of TMSCs were performed using a method described previously with modification [23]. Adherent primary TMSCs were maintained in high glucose DMEM (Gibco, Grand Island, NY, USA) containing 10\% fetal bovine serum with $1 \mathrm{X}$ antibiotic-antimycotic (Gibco). TMSCs from passages 6 through 7 were used for this study. For IL1 $\beta$ priming, TMSCs were cultured in the presence of IL1 $\beta$ (10 ng/mL, Peprotech) containing medium for $24 \mathrm{~h}$. For co-culture with osteoclast, TMSCs were seeded onto the upper $0.4 \mu \mathrm{m}$ polyethylene terephthalate membrane chamber of transwell plate (Corning, Corning, NY, USA). For IL1 $\beta$ priming, TMSCs in transwell were changed with IL1 $\beta(10 \mathrm{ng} / \mathrm{mL})$ containing medium. After $24 \mathrm{~h}$, IL1 $\beta$ priming TMSCs were washed twice with PBS and used for co culture with osteoclast in the lower chamber.

BM-MSCs were purchased from Lonza and cultured in the same medium. BM-MSCs from passages 4 through 5 were used for this study and IL1 $\beta$ priming of BM-MSCs were performed in the same way as described above. 


\subsection{Multi-lineage differentiation assays of TMSCs}

To induce osteogenic or adipogenic differentiation, confluent TMSCs were incubated with osteogenesis or adipogenesis differentiation media respectively. All the reagents and differentiation kits in this study were purchased from Lonza. Calcium deposition was evaluated by Alizarin Red $\mathrm{S}$ staining after 3 weeks of osteogenic differentiation. Oil Red $\mathrm{O}$ staining was used to assess lipid droplet after 3 weeks of adipogenic differentiation. To induce chondrogenic differentiation, spherical cell pellets were cultured in chondrogenesis differentiation media for 6 weeks. Sections were counterstained with nuclear fast red and evaluated cartilage matrix deposition by Alcian blue staining. Samples were examined using light microscopy (Olympus, Tokyo, Japan).

\subsection{Osteoclast induction of RAW 264.7 and co- culture with TMSCs}

Murine monocytic cell line RAW 264.7 (ATCC, Manassas, MA, USA) was maintained in the same medium used in TMSC culture. Osteoclastogenesis of monocytes/macrophages was conducted using a method previously described with minor modification [24]. For induction into osteoclast, RAW 264.7 were cultured in TMSC culture medium with RANKL $(100 \mathrm{mg} / \mathrm{mL}$, Peprotech, Cranbury, NJ, USA) and PD98059 (20uM, Gibco). After 2 days, RAW 264.7-derived osteoclastic cells were co-cultured with TMSCs. After 1 days, osteoclast related genes were evaluated by Quantitative PCR and number of NFATc1 expressing cells was analyzed by flow cytometry analysis.

\subsection{Flow cytometry}

TMSCs were characterized using several cell surface markers by flow cytometry. Cell surface staining was performed with fluorescent conjugated antibodies for 30 min at $4{ }^{\circ} \mathrm{C}$ in the dark chamber. All antibodies used for surface staining were purchased from eBioscience and $\mathrm{BD}$ Pharmigene. RAW 264.7-derived osteoclasts were fixed and permeabilized using BD Cytofix/Cytoperm Solution Kit (BD Biosciences, Bedford, MA, USA) and stained with PE-conjugated NFATc1 antibody (Biolegned). All sample were analyzed with FACS-LSR Fortessa (BD Bioscience) and data were analyzed with FlowJo software.

\subsection{CFSE proliferation assay}

Human peripheral blood mononuclear cells (PBMC) were purchased from ALLCELL Technologies. PBMCs were labelled with $3 \mu \mathrm{M}$ carboxyfluorescein succinimidyl ester
(CFSE, Thermo Fisher) for $4 \mathrm{~min}$ at room temperature and was washed twice with culture medium. Co-culture was established by incubating $6 \times 10^{5}$ PBMCs and $3 \times 10^{4}$ TMSCs per well in 24-well plates with culture medium (RPMI-1640 medium supplemented with $10 \%$ fetel bovine serum, $1 \%$ antibiotic antimycotic) for 6 days. PHA-M was added to stimulate $\mathrm{T}$ cell proliferation. Flow cytometry analysis for cell division by dilution of CFSE was conducted to assess PBMC proliferation.

\subsection{Real-time PCR}

Total RNA was extracted using RNeasy plus kit (Qiagen,, Valencia, CA, USA) and was transcribed to cDNA using Maxima First Strand cDNA Synthesis Kit (Thermo Fisher Scientific, Waltham, MA, USA). Quantitative PCR was performed using QuantStudio ${ }^{\text {TM }} 5$ Real-Time PCR System (Applied Biosystems) with TB Green ${ }^{\circledR}$ Premix Ex Taq ${ }^{\mathrm{TM}}$ II master mix (Takara, Kyoto, Japan). Gene specific primers sequences are listed in supplement Table S1.

\subsection{Enzyme-linked immunosorbent assay (ELISA)}

TMSCs conditioned medium was analyzed by ELISA according to the manufacturer's instructions for human OPG (Thermo Fisher Scientific) and human IL6 (R\&D system, Minneapolis, MN, USA). Blood was collected from venous cava under general anesthesia and clotted at room temperature for $2 \mathrm{~h}$. Serum was preserved at $80{ }^{\circ} \mathrm{C}$ until analysis. Serum analysis was performed according to the manufacturer's instructions for mouse RANKL (R\&D systems), mouse OPG (R\&D systems), mouse Gla-osteocalcin (Takara), and mouse CTX-1 (LSBio, Seattle, WA, USA).

\subsection{Western blot analysis}

TMSCs were washed twice with PBS and lysed in RIPA lysis buffer supplemented with cocktail of protease/phosphatase inhibitors (Thermo Fisher Scientific). Supernatants were collected after centrifugation at $12,000 \mathrm{rpm}$ for $10 \mathrm{~min}$. Humerus was frozen rapidly using liquid nitrogen immediately after dissection. Frozen humerus was homogenized using a mortar and pestle on dry ice. Whole protein of bone was extracted in ice by RIPA lysis buffer supplemented with cocktail of protease/phosphatase inhibitors for $20 \mathrm{~min}$. Supernatants were collected after centrifugation at $12,000 \mathrm{rpm}$ for $10 \mathrm{~min}$ and protein concentration was measured using BCA protein assay kit (Pierce). Primary antibodies used were anti-Wnt16 (Thermo Fisher Scientific), anti-RUNX2, anti-osteocalcin, anti-CathepsinK (Santa Cruz Biotechnology Inc., Dallas, TX, USA), and anti-beta actin (Sigma-Aldrich) antibodies. 
Secondary antibodies used were HRP conjugated antibodies. Protein bands were visualized with $\mathrm{ECL}^{\mathrm{TM}}$ Prime Western Blotting Detection Reagent (Amersham) in GBOX chemi XX9 (Syngene).

\subsection{Histological analysis}

For histological analysis, isolated femurs from euthanatized mice were fixed with $0.1 \mathrm{M}$ phosphate buffer containing $4 \%$ paraformaldehyde (Sigma-Aldrich) at $4{ }^{\circ} \mathrm{C}$ for a 3 days. Then they were decalcified in $14.5 \%$ EDTA solution (sigma) for 1 weeks at room temperature. Decalcified tissues were rinsed, dehydrated, and paraffin embedded in accordance with general protocols. Paraffinized specimens were sectioned in $4 \mu \mathrm{m}$ thickness with a microtome. Histological study was performed with Harris hematoxylin (BBC Biochemical, Vernon, WA, USA) and Eosin Y (BBC Biochemical). Images were obtained by inverted Nikon TS2R-FL microscope (Nikon).

\subsection{Micro CT measurement}

Evaluation of trabecular structure in distal femoral bones was performed using micro-computed tomography (microCT) (Skyscan 1072, Kontich, Belgium). Fixed distal femurs in 4\% PFA were scanned at $14.85 \mu \mathrm{m}$ pixel size with $50 \mathrm{kVp}, 200 \mu \mathrm{A}, 0.5 \mathrm{~mm}$ AI filter. Data were reconstructed using NRecon software and Tomographic image were analyzed by CTscan software (ver 1.6).

\subsection{Statistical analysis}

Data from quantitative experiments are represented as means standard error of the mean. One way Anova or T-test with Bonferroni Correction was calculated using Graphpad prism 5 software. For all tests, a significance level of 5\% was assigned.

\section{Results}

\subsection{IL1ß priming TMSC expresses bone homeostasis-related components}

BM-MSCs-treated with IL $1 \beta$ at low concentration $(0.1 \mathrm{ng} /$ $\mathrm{mL}$ ) had enhanced differentiation potential into osteoblast [22]. Nevertheless, it is not well understood whether pre- or continuous treatment of IL1 $\beta$ increase differentiation potential of TMSCs to osteoblast. Therefore, we examined whether pre-treatment with IL1 $\beta$ for 1 day (IL1 $\beta$ priming) before induction of osteogenic differentiation enhances osteogenic potential of TMSCs.
TMSCs-treated with IL1 $\beta$ for 1 day had no difference in its morphology and MSC surface CD marker expression profile compared to control (Supplementary Fig. 1, Fig. 1A). However, our preliminary RNAseq analysis showed that pro-osteogenic transcription factors and signaling factors such as RUNX2 (1.48 fold), TWIST 2 (1.79 fold), IGF2 (3.5 fold), BMP2 (12.66 fold), BMP7 (6.55 fold), WNT10A (2.03 fold), and WNT16 (2.88 fold) were differentially increased in IL1 $\beta$ priming TMSCs compared to mock control (Data not shown). In comparison with mock TMSCs, IL1 $\beta$ priming TMSCs produced more alizarin red $\mathrm{S}$ stained osteoblasts after osteogenic induction for 21 days but did not efficiently produce Alcian blue stained chondrocytes when inducing chondrogenic differentiation (Fig. 1B). However, there was no difference in adipogenic differentiation potential between mock and IL1 $\beta$ priming TMSCs (Fig. 1).

OPG secreted by osteoblasts in bone was able to prohibit excessive bone resorption $[25,26]$. IL6 is an immunomodulatory cytokine that can be induced by IL1 $\beta$ signaling in MSC [27]. As predicted, high expression and secretion of IL6 but no significant increase in the secretion of OPG was observed in IL1 $\beta$ priming TMSCs compared to mock TMSCs (Fig. 1C, D). Interestingly, expression of Wnt 16 was significantly increased in IL1 $\beta$ priming TMSCs (Fig. 1D). These results suggest that IL1 $\beta$ priming TMSCs have enhanced osteogenic differentiation potential and secrete components such as IL6 and Wnt16 that are able to regulate osteoclast and osteoblast differentiation in the bone.

\subsection{IL1ß priming TMSCs decrease osteoclast related gene expression in RANKL-induced differentiation}

We confirmed that IL6 decreased expression of osteoclast genes such as c-fos and NFATc1 under the RANKL concentration of $100 \mathrm{ng} / \mathrm{ml}$ in RAW 264.7-derived osteoclasts (Supplementary Fig. 2). However, expression of cathepsin $\mathrm{K}$ (ctsk) was decreased only in the presence of OPG $(50 \mathrm{ng} / \mathrm{mL})$. Interestingly, treatment of both IL6 and OPG synergistically suppressed expression of osteoclast genes (Supplementary Fig. 2). As shown in Fig. 1C, D, IL1 $\beta$ priming TMSCs was able to secrete OPG and IL6. Therefore, we hypothesized that IL1 $\beta$ priming TMSCs may also suppress osteoclast differentiation by secretion of OPG and IL6.

To see whether IL1 $\beta$ priming TMSCs suppress osteoclast differentiation, IL1 $\beta$ priming TMSCs were co-cultured with RAW 264.7-derived osteoclasts. IL1 $\beta$ priming TMSC significantly decreased the RAW 264.7 cell population that strongly expressed NFATc1 (Fig. 2). Furthermore, IL1 $\beta$ priming TMSCs not only suppressed 
A
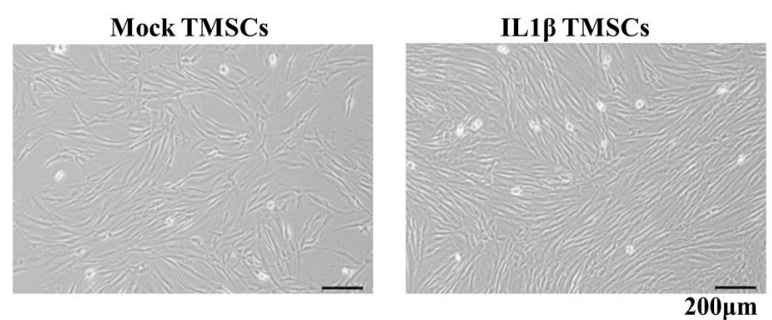

B

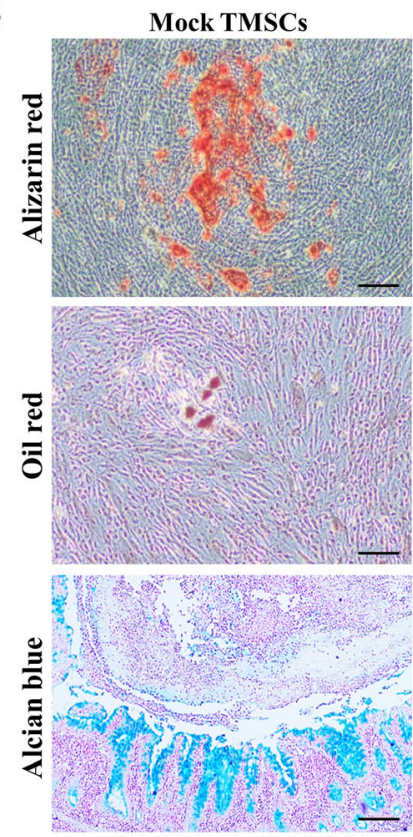

C
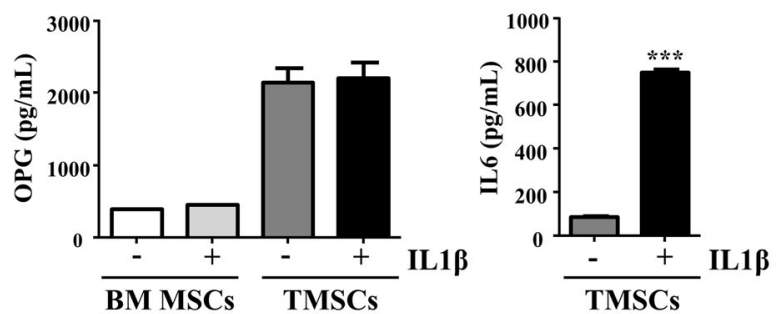

D
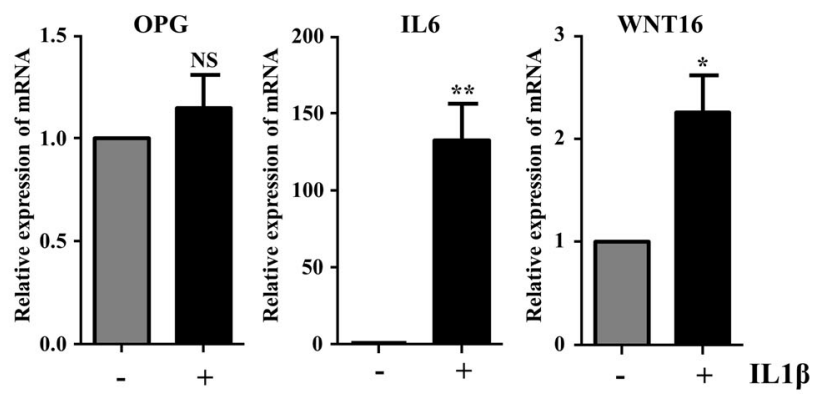

$\mathbf{E}$

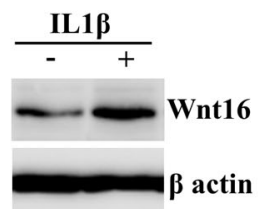

D. Quantitative PCR analysis of mRNA expression level of OPG, IL6, and WNT16 in TMSCs. E. Western blot analysis of Wnt16 expression in mock and IL1 $\beta$ priming TMSC lysates. $* p<0.05$ $* * p<0.01 * * * p<0.001$ compared with Mock TMSCs

division. 6 days after PHA stimulation (Day 6), CFSE low PBMC population was decreased about $10 \%$ when they were co-cultured with IL1 $\beta$ priming TMSCs compared to that were co-cultured with mock TMSCs (Fig. 3A).

In addition, at Day 1, RANKL expression in all the CD4 positive and CD4 negative cells was decreased about $8.3 \%$ and $15 \%$, respectively when co-cultured with IL $1 \beta$ priming TMSCs. However, mock TMSC did not affect RANKL expression in PHA-stimulated PBMCs. (Fig. 3B). These results suggested that IL1 $\beta$ priming TMSCs have immunomodulatory capacity to regulate immune cells in osteoporotic development.

To investigate immunomodulatory function of mock and IL1 $\beta$ priming TMSC, TMSCs were co-cultured with PBMCs that had been labeled with CFSE to track cell 
A
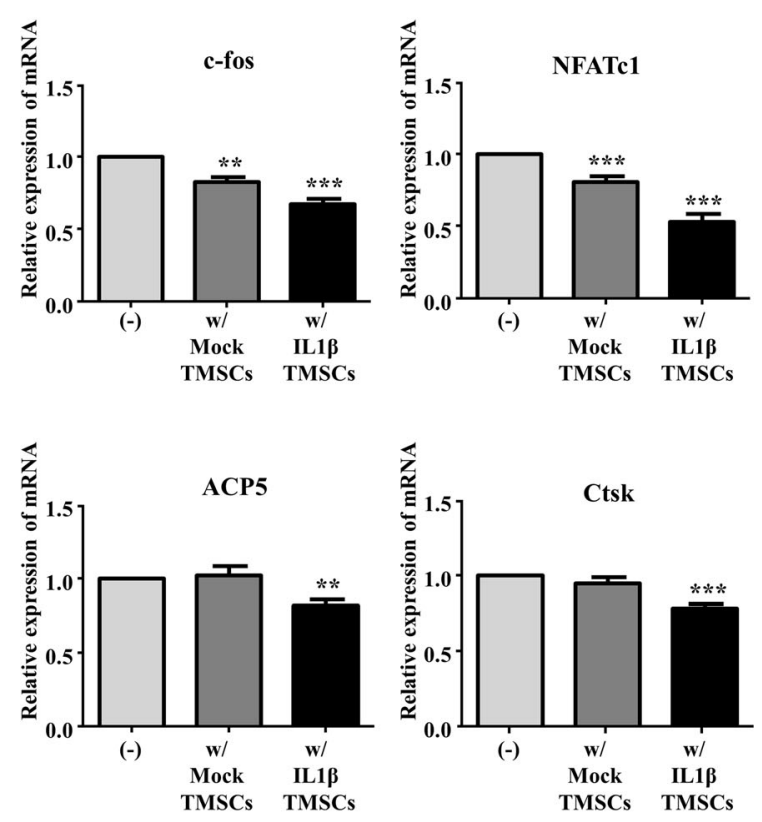

Fig. 2 Effects of IL1 $\beta$ priming TMSCs on RAW 264.7 cell-derived osteoclast differentiation. A Quantitative PCR analysis of mRNA expression levels of the osteoclast markers in osteoclasts co-cultured

\subsection{Treatment of IL1ß priming TMSCs increases bone mineral density in OVX mice}

To study whether IL1 $\beta$ priming TMSCs have therapeutic potential in osteoporosis, we established an animal model for osteoporosis by surgical removal of ovaries in 7 weeks old female mice followed by low calcium diet to enhance bone loss (OVX model). 8 weeks after ovariectomy, we confirmed osteoporotic features in the bone of OVX mice such as significant loss of trabecular bone volume due to significant decrease of trabecular thickness, BV/TV, and trabecular number (supplementary Fig. 3). At this point, IL1 $\beta$ priming TMSCs or mock TMSCs were intravenously injected into the OVX mice (twice, 4 weeks apart). Four weeks after the last administration, all mice were sacrificed, and bone tissues were examined. (Fig. 4A).

As shown in representative micro CT images in Fig. 4B, structural impairment of trabecular microarchitecture in control OVX mice was improved in IL1 $\beta$ priming TMSCtreated OVX mice compared with mock TMSC-treated OVX or vehicle-treated OVX mice. Corresponding to the micro CT image analysis, histological examination of H\&E staining sections from proximal femurs also showed that number of trabecular in IL1 $\beta$ priming TMSC-treated OVX mice was increased compared to vehicle or mock TMSCtreated OVX mice (Fig. 4C).
B

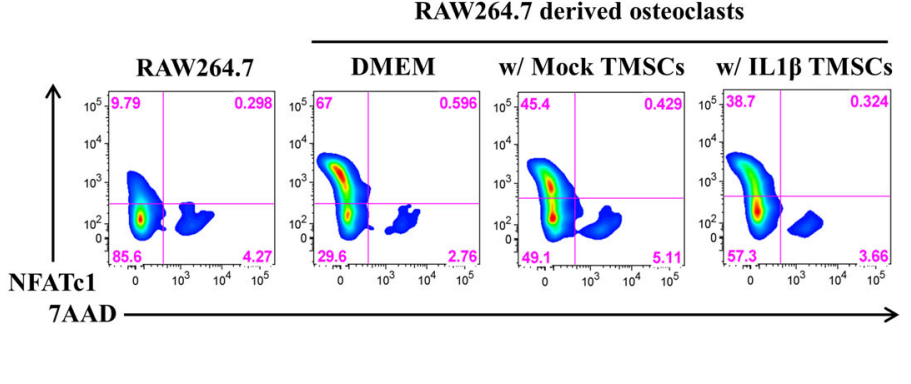

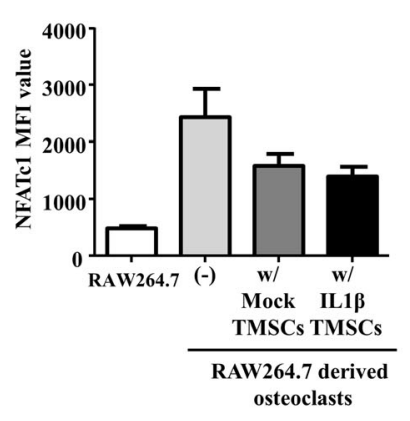

with Mock or IL1 $\beta$ priming TMSCs. B Representative FACS images of NFATc1 expression in osteoclasts co-cultured with Mock or IL1 $\beta$ priming TMSCs

In quantitative analysis of micro $\mathrm{CT}$ images, Trabecular $\operatorname{BMD}(p=0.004), \mathrm{BV} / \mathrm{TV}(p=0.02)$, and trabecular numbers $(p=0.008)$ were significantly increased, but trabecular separation $(P=0.006)$ was significantly decreased in IL1 $\beta$ priming TMSC-treated OVX mice compared with vehicle-treated OVX mice (Fig. 4D). However, trabecular thickness was decreased in all the OVX mice compared with normal mice, and was not recovered in IL1 $\beta$ priming TMSC-treated OVX group. Overall, treatment of mock TMSC did not show significant changes compared to vehicle-treated OVX mice. (Fig. 4D). These results suggested that IL1 $\beta$ priming TMSCs have therapeutic potential to improve estrogen deficiency induced osteoporosis.

\subsection{Treatment of IL1ß priming TMSCs restores both bone formation and resorption markers in OVX mice}

To examine systemic effects of IL1 $\beta$ priming TMSCs in OVX mice, blood serum was analyzed. OVX mice had relatively high RANKL/OPG, an index of osteoclastogenic stimulation $[28,29]$, compared with that of normal mice. However, in IL1 $\beta$ priming TMSC-treated OVX mice, serum RANKL/OPG ratio was significantly decreased on the first day of administration compared to sham control or vehicle-treated OVX mice, but this difference was not significant on 14 days after intravenous administration of 
A

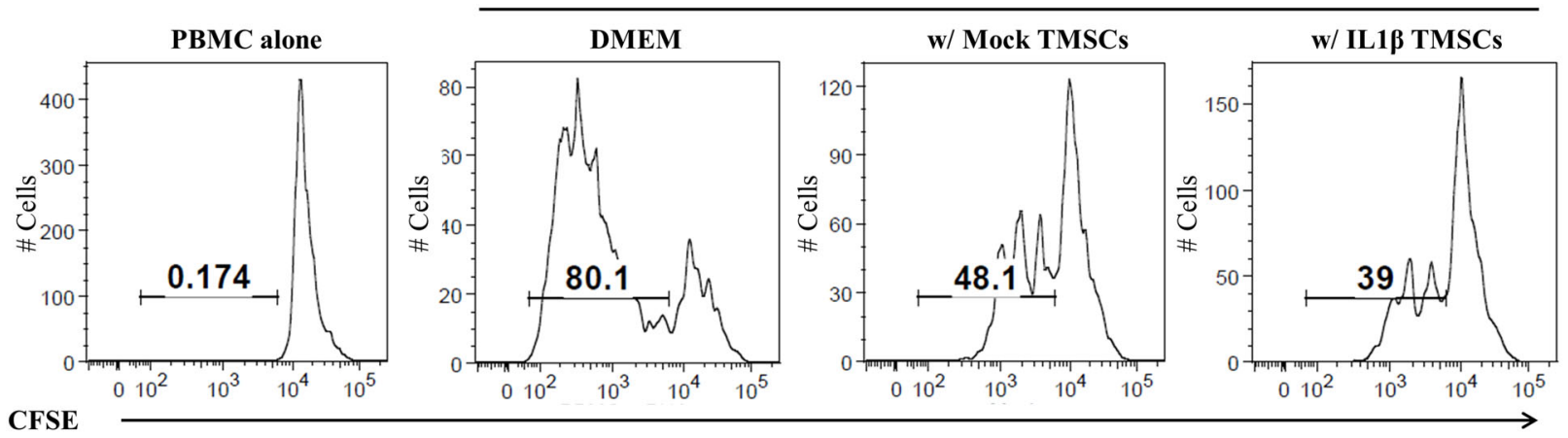

B
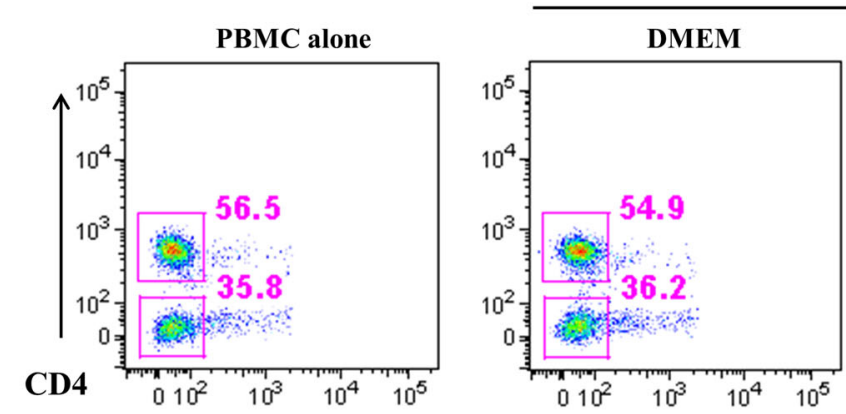

PBMC + PHA 1d

\section{AAD}
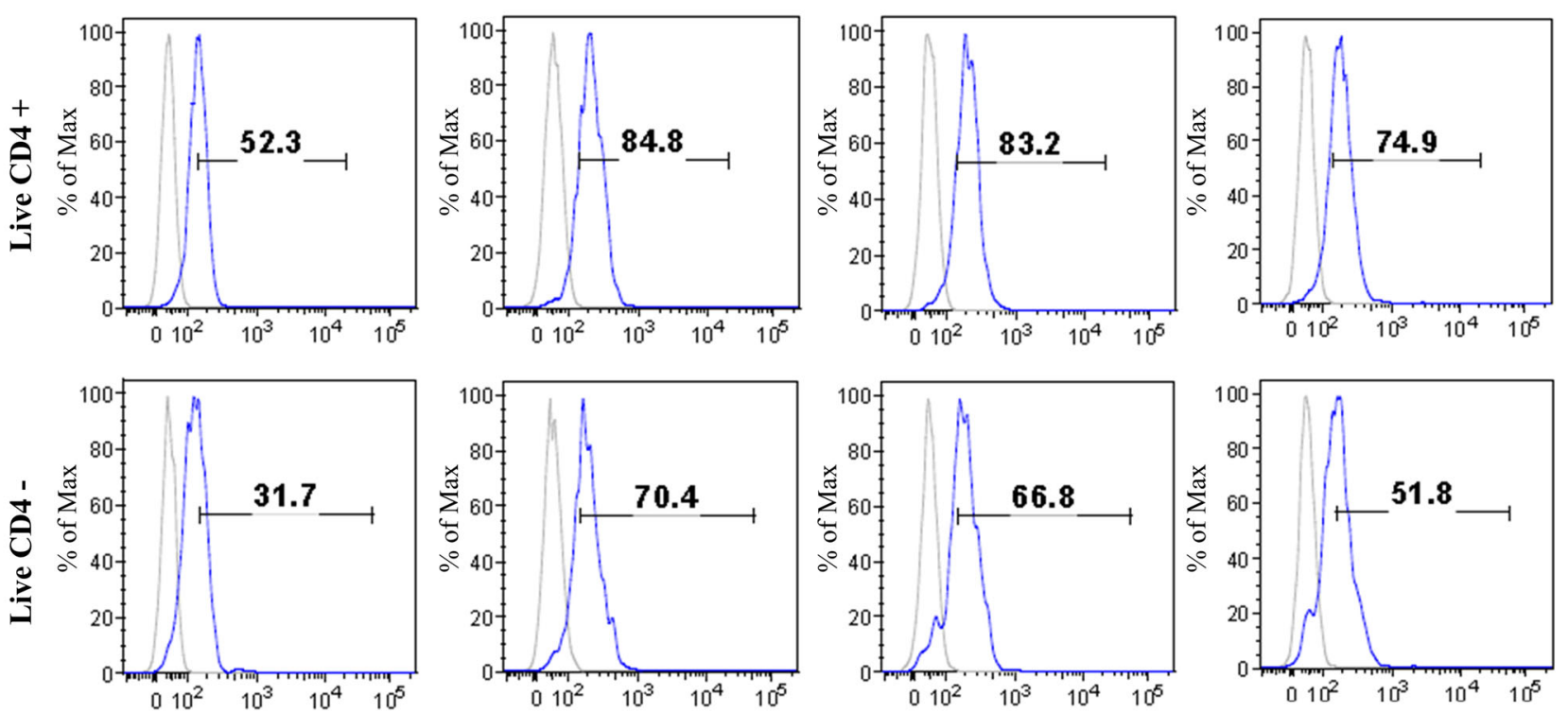

\section{RANKL}

Fig. 3 Immunomodulatory effects of IL $1 \beta$ priming TMSCs on PHAstimulated PBMCs. A Representative FACS images of proliferation of PBMC co-cultured with Mock or IL1 $\beta$ priming TMSCs.

IL1 $\beta$ priming TMSCs (Fig. 5A, B). 4 weeks after second administration of vehicle or IL priming TMSCs, serum RANKL/OPG ratio was similar level in all OVX mice group. Thus, these results suggested that serum RANKL/
B Representative FACS images of RANKL expression on PHAstimulated PBMCs co-cultured with Mock or IL1 $\beta$ priming TMSCs

OPG ratio was temporally regulated immediately after systemic infusion of IL1 $\beta$ priming TMSCs.

16 weeks after ovariectomy, both bone formation marker, Gla-osteocalcin, and bone resorption marker, CTX-1, were reduced in vehicle-treated OVX mice compared to 
A

\begin{tabular}{|c|c|c|c|}
\hline \begin{tabular}{|c|}
$7 \mathrm{~W}$ \\
old mice
\end{tabular} & $8 \mathrm{~W}$ & $4 \mathrm{~W}$ & $4 \mathrm{~W}$ \\
\hline $\begin{array}{c}\uparrow \\
\text { ovx }\end{array}$ & & & \\
\hline
\end{tabular}

B

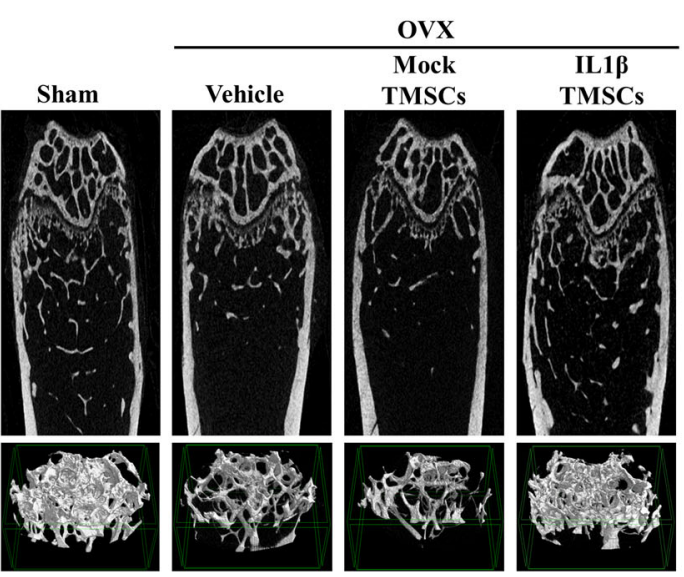

C

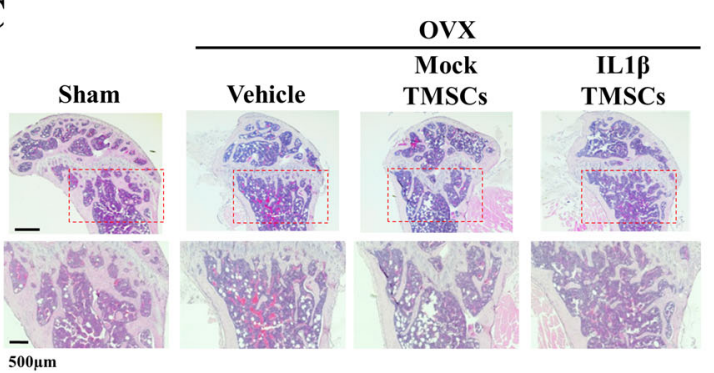

D
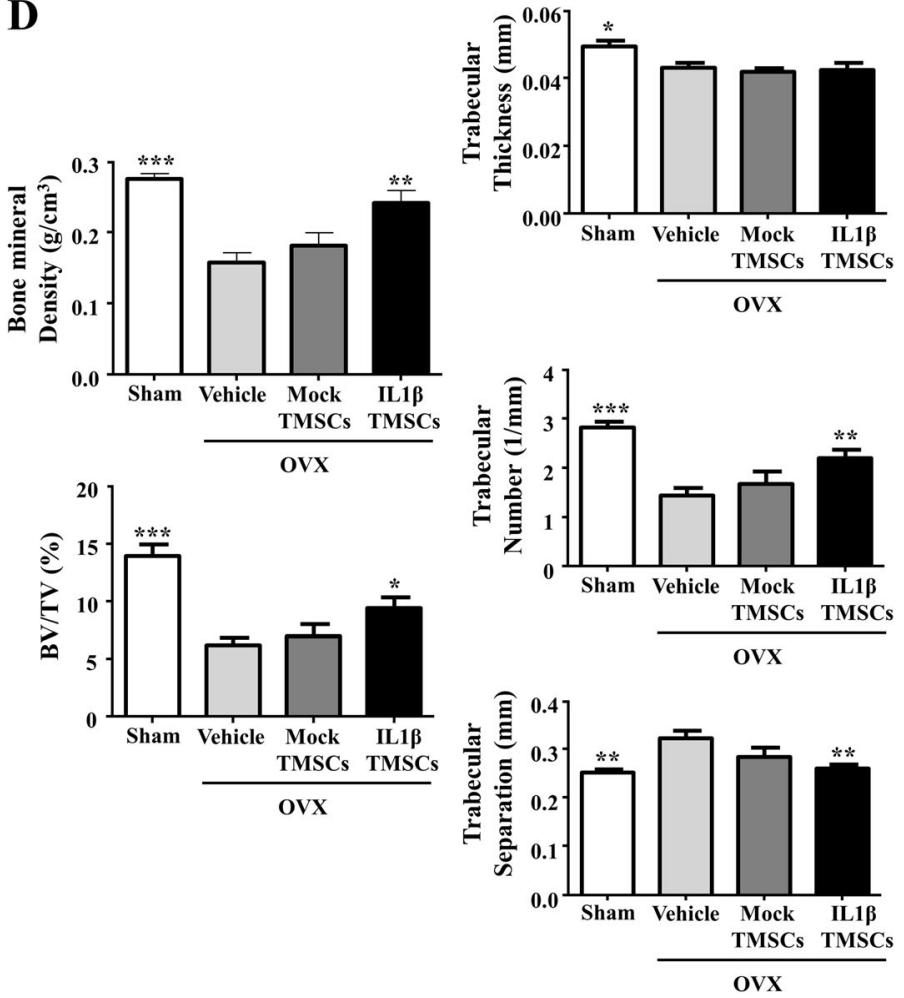

Fig. 4 Therapeutic effects of systemic infusion of IL1 $\beta$ priming TMSCs in ovariectomized osteoporosis mouse model. A Experiment scheme. B Representative micro CT image of the femurs harvested at 8 weeks after cells injection. C Representative H\&E stained sections

that of normal or sham mice. Serum Gla-osteocalcin in sham mice which were fed calcium diet was also high compared to that of normal mice. However, administration of IL1 $\beta$ priming TMSCs restored not only serum Gla-osteocalcin level but also serum CTX-1 level in OVX mice (Fig. 5C, D).

Corresponding to the serum analyses, protein levels of both bone formation marker (RUNX2 and osteocalcin) and resorption maker (cathepsin $\mathrm{K}$ ) were recovered in whole bone tissue of IL1 $\beta$ priming TMSC-treated OVX mice (Fig. 5E). Overall, these results suggested that IL1 $\beta$ priming TMSCs have potential to restore bone homeostasis in osteoporosis.

of femurs. D Quantitative analysis of trabecular bone parameters, including bone volume/tissue volume, bone mineral density, trabecular thickness, trabecular numbers, and trabecular separation. $* p<0.05 * * p<0.01 * * * p<0.001$ compared with OVX-vehicle

\section{Discussion}

Stem cell therapy that can ensure the constant therapeutic efficacy to rebalance bone homeostasis are mandatory for the successful treatment of osteoporosis. However, administration of patient's own MSCs or MSCs from alternative sources still exhibited a short-term engraftment and limited efficacy to rebalance bone formation and resorption in osteoporosis $[24,30]$. Human palatine tonsils can serve as an alternative source for MSCs [23]. Human tonsil tissue can be obtained from tonsillectomy and average amount of adherent primary TMSCs obtained from $1 \mathrm{~g}$ of the tonsil tissue was about $1.3 \times 10^{7}$ at passage 0 . Number of the tonsil-derived MSCs (TMSCs) at passage 7 can reach up to $3.5 \times 10^{13}$ (unpublished data). Thus, tonsils can be a good source that are able to provide commercial scale TMSCs for cell therapy by isolation of MSCs from a single donor. 
A

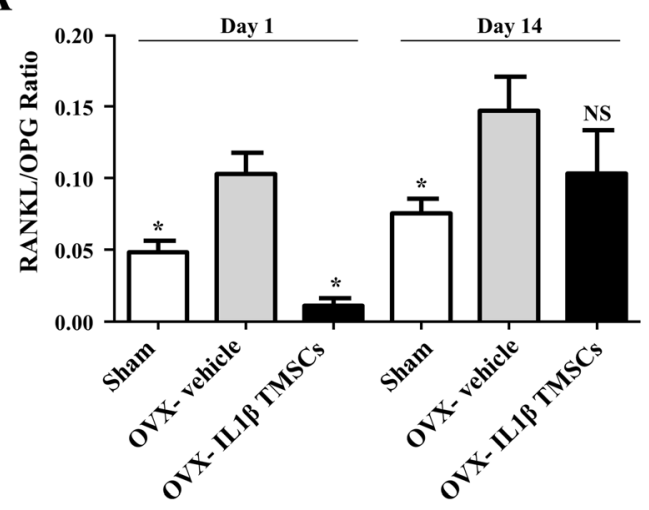

B

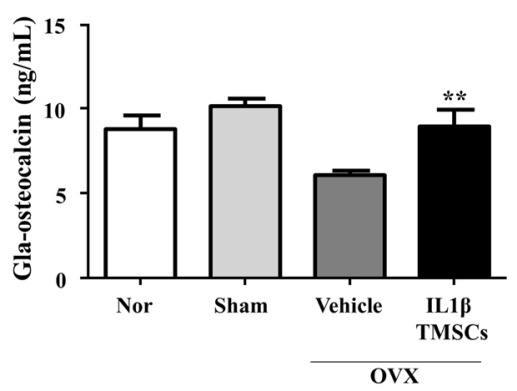

C

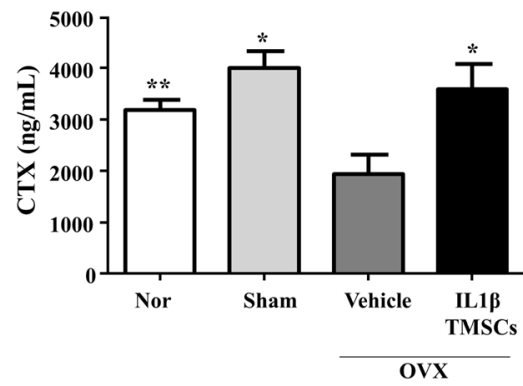

D

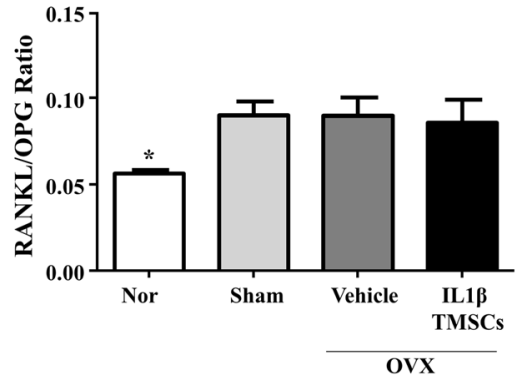

$\mathbf{E}$

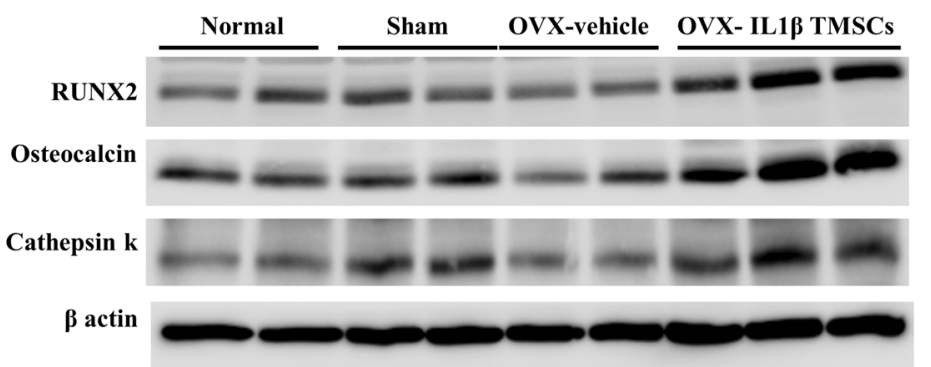

Fig. 5 Function of IL1 $\beta$ priming TMSCs for balance between bone formation and resorption markers. A RANKL/OPG ratio in serum harvested at day 1 and day 14 after IL1 $\beta$ priming TMSC infusion via ELISA assay $(\mathrm{n}=3) . * p<0.05$ compared with OVX-vehicle. BD Quantification of Gla-osteocalcin and CTX and RNAKL/OPG ratio

Recent study showed that systemic infusion of naïve TMSCs or conditioned medium in senile osteoporosis model mice showed reduction of visceral adipose tissue mass, but bone loss was not significantly recovered in these mice [11]. Interestingly, intra-tibial injection of naïve TMSCs in ovariectomized postmenopausal osteoporosis model mice improved bone mineral density compared to untreated animals [12]. Moreover, subcutaneous injection of TMSCs-embedded hydrogel led to better recovery of the femoral heads in ovariectomized postmenopausal osteoporosis model mice [13]. These results suggest that local administration of TMSCs has therapeutic benefits for osteoporosis. However, therapeutic potential of systemic in serum harvested 8 weeks after IL1 $\beta$ priming TMSC injection ( $\mathrm{n}=5$ or 6 ) using ELISA assay. $* p<0.05 * * p<0.01$ compared with OVX-vehicle. E Western Blot analysis of whole humerus lysates $(\mathrm{n}=2$ or 3$)$

infusion of naïve or priming TMSCs for postmenopausal osteoporosis has not been demonstrated yet.

In this study, we investigated the therapeutic potential of systemic infusion of TMSCs primed by an inflammatory cytokine (IL-1 $\beta$ ) that mimic inflammation increased by the loss of estrogens at menopause [19, 31]. TMSCs expressed typical MSC surface marker such as CD73, CD90 and CD105 and expression of the markers was not changed after IL1 $\beta$ priming. However, IL1 $\beta$ priming TMSCs suppressed proliferation of PHA-stimulated PBMCs better than mock TMSCs. In addition, RANKL expression in PHA-stimulated PBMCs was also efficiently suppressed by IL1 $\beta$ priming TMSCs compared to mock TMSCs. These 
results support that anti-inflammatory effects of TMSCs are enhanced by IL1 $\beta$ priming.

OPG is largely expressed by osteoblast lineage cells of bone and may prevent unnecessary bone resorpion by acting as a decoy receptor to inhibit RANK-RANKL binding in osteoclast differentiation $[25,26]$. It has been shown that TMSCs expressed significantly higher levels of OPG compared to bone marrow or adipose derived mesenchymal stem cells [16]. Our results also support higher OPG secretion in TMSCs compared to BM-MSCs. IL6 is a multifunctional cytokine which coordinates not only immune responses but also bone homeostasis [32-34]. In fact, IL6 has dual functions either as a negative or positive effector on osteoclastogenesis $[35,36]$. It is well known that IL6 expression can be enhanced by IL1 $\beta$ priming in MSCs. Although IL6 modulates RANKL expression in osteoblasts and indirectly promotes functional activity and maturation of RANKL-induced osteoclasts in the existence of soluble receptor (sIL-6R), many studies also showed that IL6 may directly inhibit RANKL-induced osteoclast differentiation [36-38].

Osteoclast differentiation genes such as c-fos, NFATc1, ACP5, and Ctsk that are related with RAW 264.7-derived osteoclast formation were suppressed by IL1 $\beta$ priming TMSCs compared to mock TMSC. In particular, NFATc1, a key master transcription factor of osteoclast differentiation, was downregulated to the protein level in undifferentiated RAW 264.7. Furthermore, expression of c-fos and NFATc1 was inversely proportional to the concentration of IL6, and co-treatment of OPG with IL6 enhanced the IL6 mediated inhibition of c-fos and NFACTc1 in our osteoclast assay. Thus, these results suggest that high levels of inherent OPG secretion and IL1 $\beta$ induced IL6 secretion in IL1 $\beta$ priming TMSCs may promote inhibition of osteoclast gene expression and RANKL expression of immune cells.

Interestingly, expression of Wnt16, a positive regulator of cortical and trabecular bone mass and structure [39, 40], was also increased in IL1 priming TMSCs. Previous studies demonstrated that Wnt16 increased OPG expression in osteoblasts and regulated osteoclast formation in conjunction with Wnt5a [40, 41]. In addition, overexpression of Wnt16 increased mainly trabecular bone mass in vivo $[40,42]$. For this reason, we hypothesized that IL1 $\beta$ priming TMSCs have constant and enhanced therapeutic efficacy in osteoporosis.

In this study, IL1 $\beta$ priming TMSCs were systemically infused in ovariectomized postmenopausal osteoporosis (OVX) model mice established by combining full bilateral ovariectomy with low calcium diet to insure bone mineral loss. Two months after systemic cell infusion, IL1 $\beta$ priming TMSCs restored BMD to normal mice level, but mock TMSCs did not have significant effects on BMD in OVX mice. Six-weeks low calcium diet significantly decreased bone resorption markers such as urinary excretion of deoxypyridinoline and serum tartrate-resistance acid phosphatase [43, 44]. Interestingly, we also found that serum RANKL OPG ratio was significantly decreased in OVX mice after IL1 $\beta$ priming TMSC infusion. Serum CTX-1, an index of bone resorption, was decreased in OVX mice with low calcium diet. Infusion of IL1 $\beta$ priming TMSCs restored both serum Gla-osteocalcin, an index of bone formation, and serum CTX-1 in OVX mice compared to vehicle control. In addition, expression of RUNX2, osteocalcin, and cathepsin $\mathrm{K}$ were also increased in whole bone of IL1 $\beta$ priming TMSC injected OVX mice compared to vehicle control. These results suggest that IL1 $\beta$ priming TMSCs may lead to modulate inflammation and recover both bone formation and resorption in postmenopausal osteoporosis, which may contribute to bone homeostasis.

In conclusion, we suggested that IL1 $\beta$ priming TMSCs has therapeutic potential for postmenopausal osteoporosis, which may improve bone density, restore bone homeostasis, and enhance anti-inflammatory capacity.

Acknowledgements This study was supported by grant from the Ministry of Health \& Welfare, Republic of Korea (HI16C2207)

Author contributions Study design: MJY, SKC, DHP; Study conduct and data collection: MJY, SKC, SHS, JMK, HGP; Data interpretation: MJY, SHS, SKC, DHP; Drafting manuscript: MJY, DHP.

\section{Compliance with ethical standards}

Conflicts of interest The authors declare no competing financial interests.

Ethical statement The animal studies were performed after receiving approval of the Institutional Animal Care and Use Committee of animal research center in GC pharma (approval No. GC-18-002A).

Open Access This article is licensed under a Creative Commons Attribution 4.0 International License, which permits use, sharing, adaptation, distribution and reproduction in any medium or format, as long as you give appropriate credit to the original author(s) and the source, provide a link to the Creative Commons licence, and indicate if changes were made. The images or other third party material in this article are included in the article's Creative Commons licence, unless indicated otherwise in a credit line to the material. If material is not included in the article's Creative Commons licence and your intended use is not permitted by statutory regulation or exceeds the permitted use, you will need to obtain permission directly from the copyright holder. To view a copy of this licence, visit http://creativecommons. org/licenses/by/4.0/.

\section{References}

1. Sözen T, Özışık L, Başaran NÇ. An overview and management of osteoporosis. Eur J Rheumatol. 2017;4:46-56. 
2. Marx RE. Pamidronate (Aredia) and zoledronate (Zometa) induced avascular necrosis of the jaws: a growing epidemic. J Oral Maxillofac Surg. 2003;61:1115-7.

3. Rossouw JE, Anderson GL, Prentice RL, LaCroix AZ, Kooperberg C, Stefanick ML, et al. Risks and benefits of estrogen plus progestin in healthy postmenopausal women: principal results From the Women's Health Initiative randomized controlled trial. JAMA. 2002;288:321-33.

4. Vahle JL, Sato M, Long GG, Young JK, Francis PC, Engelhardt JA, et al. Skeletal changes in rats given daily subcutaneous injections of recombinant human parathyroid hormone (1-34) for 2 years and relevance to human safety. Toxicol Pathol. 2002;30:312-21.

5. Zullo F, Palomba S, Zupi E, Russo T, Morelli M, Cappiello F, et al. Effectiveness of presacral neurectomy in women with severe dysmenorrhea caused by endometriosis who were treated with laparoscopic conservative surgery: a 1-year prospective randomized double-blind controlled trial. Am J Obstet Gynecol. 2003; 189:5-10.

6. Chavassieux P, Chapurlat R, Portero-Muzy N, Roux JP, Garcia P, Brown JP, et al. Bone-forming and antiresorptive effects of romosozumab in postmenopausal women with osteoporosis: bone histomorphometry and microcomputed tomography analysis after 2 and 12 months of treatment. J Bone Miner Res. 2019;34:1597-608.

7. Samivel R, Kim EH, Chung YJ, Mo JH. Immunomodulatory effect of tonsil-derived mesenchymal stem cells in a mouse model of allergic rhinitis. Am J Rhinol Allergy. 2015;29:262-7.

8. Lee KE, Jung SA, Joo YH, Song EM, Moon CM, Kim SE, et al. The efficacy of conditioned medium released by tonsil-derived mesenchymal stem cells in a chronic murine colitis model. PLoS One. 2019;14:e0225739.

9. Park YS, Kim HS, Jin YM, Yu Y, Kim HY, Park HS, et al. Differentiated tonsil-derived mesenchymal stem cells embedded in Matrigel restore parathyroid cell functions in rats with parathyroidectomy. Biomaterials. 2015;65:140-52.

10. Jo YI, Kim G, Jin YM, Park YJ, Kim HS, Park YS. Intracellular remodeling and accumulation of aberrant lysosomes in differentiation of tonsil-derived mesenchymal stem cells into parathyroid-like cells. Tissue Eng Regen Med. 2017;14:411-20.

11. Kim YH, Park M, Cho KA, Kim BK, Ryu JH, Woo SY, et al. Tonsil-derived mesenchymal stem cells promote bone mineralization and reduce marrow and visceral adiposity in a mouse model of senile osteoporosis. Stem Cells Dev. 2016;25:1161-71.

12. Kim G, Jin YM, Yu Y, Kim HY, Jo SA, Park YJ, et al. Double intratibial injection of human tonsil-derived mesenchymal stromal cells recovers postmenopausal osteoporotic bone mass. Cytotherapy. 2018;20:1013-27.

13. Kim G, Park YS, Lee Y, Jin YM, Choi DH, Ryu KH, et al. Tonsil-derived mesenchymal stem cell-embedded in situ crosslinkable gelatin hydrogel therapy recovers postmenopausal osteoporosis through bone regeneration. PLoS One. 2018;13:e0200111.

14. Cho KA, Park M, Kim YH, Woo SY, Ryu KH. Conditioned media from human palatine tonsil mesenchymal stem cells regulates the interaction between myotubes and fibroblasts by IL1Ra activity. J Cell Mol Med. 2017;21:130-41.

15. Kim JY, Park M, Kim YH, Ryu KH, Lee KH, Cho KA, et al. Tonsil-derived mesenchymal stem cells (T-MSCs) prevent Th17mediated autoimmune response via regulation of the programmed death-1/programmed death ligand-1 (PD-1/PD-L1) pathway. J Tissue Eng Regen Med. 2018;12:e1022-33.

16. Cho KA, Park M, Kim YH, Ryu KH, Woo SY. Mesenchymal stem cells inhibit RANK-RANKL interactions between osteoclasts and Th17 cells via osteoprotegerin activity. Oncotarget. 2017;8:83419-31.
17. Tyagi AM, Srivastava K, Mansoori MN, Trivedi R, Chattopadhyay N, Singh D. Estrogen deficiency induces the differentiation of IL-17 secreting Th17 cells: a new candidate in the pathogenesis of osteoporosis. PLoS One. 2012;7:e44552.

18. Srivastava RK, Dar HY, Mishra PK. Immunoporosis: immunology of osteoporosis-role of T cells. Front Immunol. 2018;9:657.

19. Brincat SD, Borg M, Camilleri G, Calleja-Agius J. The role of cytokines in postmenopausal osteoporosis. Minerva Ginecol. 2014;66:391-407.

20. Ilesanmi-Oyelere BL, Schollum L, Kuhn-Sherlock B, McConnell M, Mros S, Coad J, et al. Inflammatory markers and bone health in postmenopausal women: a cross-sectional overview. Immun Ageing. 2019;16:15.

21. Ruscitti P, Cipriani P, Carubbi F, Liakouli V, Zazzeroni F, Di Benedetto $\mathrm{P}$ et al. The role of IL-1beta in the bone loss during rheumatic diseases. Mediators Inflamm. 2015;2015:782382.

22. Sonomoto K, Yamaoka K, Oshita K, Fukuyo S, Zhang X, Nakano $\mathrm{K}$, et al. Interleukin-1beta induces differentiation of human mesenchymal stem cells into osteoblasts via the Wnt-5a/receptor tyrosine kinase-like orphan receptor 2 pathway. Arthritis Rheum. 2012;64:3355-63.

23. Ryu KH, Cho KA, Park HS, Kim JY, Woo SY, Jo I, et al. Tonsilderived mesenchymal stromal cells: evaluation of biologic, immunologic and genetic factors for successful banking. Cytotherapy. 2012;14:1193-202.

24. Saito A, Nagaishi K, Iba K, Mizue Y, Chikenji T, Otani M, et al. Umbilical cord extracts improve osteoporotic abnormalities of bone marrow-derived mesenchymal stem cells and promote their therapeutic effects on ovariectomised rats. Sci Rep. 2018;8:1161.

25. Udagawa N, Takahashi N, Yasuda H, Mizuno A, Itoh K, Ueno Y, et al. Osteoprotegerin produced by osteoblasts is an important regulator in osteoclast development and function. Endocrinology. 2000;141:3478-84.

26. Oshita K, Yamaoka K, Udagawa N, Fukuyo S, Sonomoto K, Maeshima K, et al. Human mesenchymal stem cells inhibit osteoclastogenesis through osteoprotegerin production. Arthritis Rheum. 2011;63:1658-67.

27. Redondo-Castro E, Cunningham C, Miller J, Martuscelli L, Aoulad-Ali S, Rothwell NJ, et al. Interleukin-1 primes human mesenchymal stem cells towards an anti-inflammatory and protrophic phenotype in vitro. Stem Cell Res Ther. 2017;8:79.

28. Boyce BF, Xing L. Biology of RANK, RANKL, and osteoprotegerin. Arthritis Res Ther. 2007;9:S1.

29. Tanaka H, Mine T, Ogasa H, Taguchi T, Liang CT. Expression of RANKL/OPG during bone remodeling in vivo. Biochem Biophys Res Commun. 2011;411:690-4.

30. Zheng CX, Sui BD, Liu N, Hu CH, He T, Zhang XY, et al. Adipose mesenchymal stem cells from osteoporotic donors preserve functionality and modulate systemic inflammatory microenvironment in osteoporotic cytotherapy. Sci Rep. 2018;8:5215.

31. Malutan AM, Dan M, Nicolae C, Carmen M. Proinflammatory and anti-inflammatory cytokine changes related to menopause. Prz Menopauzalny. 2014;13:162-8.

32. Choy E, Rose-John S. Interleukin-6 as a multifunctional regulator: inflammation, immune response, and fibrosis. J Scleroderma Relat Disord. 2017;2:S1-5.

33. Blanchard F, Duplomb L, Baud'huin M, Brounais B. The dual role of IL-6-type cytokines on bone remodeling and bone tumors. Cytokine Growth Factor Rev. 2009;20:19-28.

34. Smith JK. IL-6 and the dysregulation of immune, bone, muscle, and metabolic homeostasis during spaceflight. NPJ Microgravity. 2018;4:24.

35. Kudo O, Sabokbar A, Pocock A, Itonaga I, Fujikawa Y, Athanasou NA. Interleukin-6 and interleukin-11 support human 
osteoclast formation by a RANKL-independent mechanism. Bone. 2003;32:1-7.

36. Duplomb L, Baud'huin M, Charrier C, Berreur M, Trichet V, Blanchard F, et al. Interleukin-6 inhibits receptor activator of nuclear factor kappaB ligand-induced osteoclastogenesis by diverting cells into the macrophage lineage: key role of Serine 727 phosphorylation of signal transducer and activator of transcription 3. Endocrinology. 2008;149:3688-97.

37. Yoshitake F, Itoh S, Narita H, Ishihara K, Ebisu S. Interleukin-6 directly inhibits osteoclast differentiation by suppressing receptor activator of NF-kappaB signaling pathways. J Biol Chem. 2008;283:11535-40.

38. Feng W, Liu H, Luo T, Liu D, Du J, Sun J, et al. Combination of IL-6 and sIL-6R differentially regulate varying levels of RANKL-induced osteoclastogenesis through NF-kappaB, ERK and JNK signaling pathways. Sci Rep. 2017;7:41411.

39. Martínez-Gil N, Roca-Ayats N, Monistrol-Mula A, García-Giralt N, Díez-Pérez A, Nogués X, et al. Common and rare variants of WNT16, DKK1 and SOST and their relationship with bone mineral density. Sci Rep. 2018;8:10951.

40. Movérare-Skrtic S, Henning P, Liu X, Nagano K, Saito H, Börjesson AE, et al. Osteoblast-derived WNT16 represses osteoclastogenesis and prevents cortical bone fragility fractures. Nat Med. 2014;20:1279-88.

41. Kobayashi Y, Thirukonda GJ, Nakamura Y, Koide M, Yamashita $\mathrm{T}$, Uehara $\mathrm{S}$, et al. Wnt16 regulates osteoclast differentiation in conjunction with Wnt5a. Biochem Biophys Res Commun. 2015;463:1278-83.

42. Movérare-Skrtic S, Wu J, Henning P, Gustafsson KL, Sjögren K, Windahl $\mathrm{SH}$, et al. The bone-sparing effects of estrogen and WNT16 are independent of each other. Proc Natl Acad Sci U S A. 2015;112:14972-7.

43. Bae YJ, Kim MH. Calcium and magnesium supplementation improves serum OPG/RANKL in calcium-deficient ovariectomized rats. Calcif Tissue Int. 2010;87:365-72.

44. Kim C, Park D. The effect of restriction of dietary calcium on trabecular and cortical bone mineral density in the rats. J Exerc Nutrition Biochem. 2013;17:123-31.

Publisher's Note Springer Nature remains neutral with regard to jurisdictional claims in published maps and institutional affiliations. 